\title{
DIVERGENT COINDEX SEQUENCE FOR DYNAMICAL SYSTEMS
}

\author{
RUXI SHI AND MASAKI TSUKAMOTO
}

\begin{abstract}
When a finite group freely acts on a topological space, we can define its index and coindex. They roughly measure the size of the given action. We explore the interaction between this index theory and topological dynamics. Given a fixed-point free dynamical system, the set of $p$-periodic points admits a natural free action of $\mathbb{Z} / p \mathbb{Z}$ for each prime number $p$. We are interested in the growth of its index and coindex as $p \rightarrow \infty$. Our main result shows that there exists a fixed-point free dynamical system having the divergent coindex sequence. This solves a problem posed by [TTY20].
\end{abstract}

\section{INTRODUCTION}

1.1. Background on $\mathbb{Z}_{p}$-index. Let $p$ be a prime number. When the finite group $\mathbb{Z}_{p}:=\mathbb{Z} / p \mathbb{Z}$ freely acts on a topological space, we can define its index. The $\mathbb{Z}_{p}$-index roughly measures the size of the given $\mathbb{Z}_{p}$-space. It has several astonishing applications to combinatorics [Mat03].

Tsutaya, Yoshinaga and the second-named author [TTY20] found an application of the $\mathbb{Z}_{p}$-index theory to topological dynamics. (One of their motivations is to solve a problem about the marker property of dynamical systems. This will be briefly explained in §2.2.) The purpose of this paper is to continue this investigation. In particular we solve a problem posed by [TTY20].

First we prepare terminologies of $\mathbb{Z}_{p}$-index, following the book of Matoušek [Mat03]. A pair $(X, T)$ is called a $\mathbb{Z}_{p}$-space if $X$ is a topological space and $T: X \rightarrow X$ is a homeomorphism with $T^{p}=$ id. It is said to be free if $T^{a} x \neq x$ for all $1 \leq a \leq p-1$ and $x \in X$. Since $p$ is a prime number, this condition is equivalent to the condition that $T x \neq x$ for all $x \in X$.

Let $n \geq 0$ be an integer. A free $\mathbb{Z}_{p^{-}}$space $(X, T)$ is called an $E_{n} \mathbb{Z}_{p^{-}}$ space if it satisfies:

- $X$ is an $n$-dimensional finite simplicial complex and $T$ is a simplicial map (i.e. sending each simplex to simplex affinely).

2020 Mathematics Subject Classification. 37B02, 55M35.

Key words and phrases. $\mathbb{Z}_{p}$-space, $\mathbb{Z}_{p^{-}}$-index, $\mathbb{Z}_{p}$-coindex, dynamical system, periodic point, marker property.

M.T. was supported by JSPS KAKENHI 18K03275. 
- $X$ is $(n-1)$-connected i.e., $\pi_{k}(X)=0$ for all $0 \leq k \leq n-1$.

For example, $\mathbb{Z}_{p}$ itself (with the natural $\mathbb{Z}_{p}$-action) is an $E_{0} \mathbb{Z}_{p}$-space. (We consider that $\mathbb{Z}_{p}$ is $(-1)$-connected.) The join ${ }^{1}$ of the $(n+1)$ copies of $\mathbb{Z}_{p}$

$$
\left(\mathbb{Z}_{p}\right)^{*(n+1)}=\underbrace{\mathbb{Z}_{p} * \cdots * \mathbb{Z}_{p}}_{(n+1) \text { times }}
$$

is an $E_{n} \mathbb{Z}_{p}$-space. Here $\mathbb{Z}_{p}$ acts on each component of $\left(\mathbb{Z}_{p}\right)^{*(n+1)}$ simultaneously.

An $E_{n} \mathbb{Z}_{p}$-space is not unique. But they are essentially unique for our purpose here [Mat03, Lemma 6.2.2]: If $(X, T)$ and $(Y, S)$ are both $E_{n} \mathbb{Z}_{p}$-spaces then there are equivariant continuous maps $f: X \rightarrow Y$ and $g: Y \rightarrow X$.

Let $(X, T)$ be a free $\mathbb{Z}_{p}$-space. We define its index and coindex by $\operatorname{ind}_{p}(X, T):=\min \left\{n \geq 0: \exists\right.$ an equivariant continuous $\left.: X \rightarrow E_{n} \mathbb{Z}_{p}\right\}$, $\operatorname{coind}_{p}(X, T):=\max \left\{n \geq 0: \exists\right.$ an equivariant continuous $\left.: E_{n} \mathbb{Z}_{p} \rightarrow X\right\}$.

We set $\operatorname{ind}_{p}(X, T)=\infty$ if there is no equivariant continuous map from $X$ to $E_{n} \mathbb{Z}_{p}$ for any $n \geq 0$. We use the convention that $\operatorname{ind}_{p}(X, T)=$ $\operatorname{coind}_{p}(X, T)=-1$ for $X=\emptyset$. We sometime abbreviate $\operatorname{ind}_{p}(X, T)$ $\left(\operatorname{resp} . \operatorname{coind}_{p}(X, T)\right)$ as $\operatorname{ind}_{p} X\left(\operatorname{resp} . \operatorname{coind}_{p} X\right)$.

It is known that if there exists an equivariant continuous map from $E_{m} \mathbb{Z}_{p}$ to $E_{n} \mathbb{Z}_{p}$ then $m \leq n$ (see [Mat03, Theorem 6.2.5]). From this,

$$
\operatorname{ind}_{p}(X, T) \geq \operatorname{coind}_{p}(X, T) \text {. }
$$

Moreover, $\operatorname{ind}_{p} E_{n} \mathbb{Z}_{p}=\operatorname{coind}_{p} E_{n} \mathbb{Z}_{p}=n$.

1.2. Background on dynamical systems. A pair $(X, T)$ is called a (topological) dynamical system if $X$ is a compact metrizable space and $T: X \rightarrow X$ is a homeomorphism. (Notice that here we assume the compactness of $X$. This is essential for our result.)

Let $n \geq 1$ and $(X, T)$ a dynamical system. We define $P_{n}(X, T)$ as the set of $n$-periodic points of $(X, T)$ :

$$
P_{n}(X, T):=\left\{x \in X: T^{n} x=x\right\} .
$$

We often abbreviate this as $P_{n}(X)$.

\footnotetext{
${ }^{1}$ Recall that for two topological spaces $X$ and $Y$, the join $X * Y$ is defined by

$$
X * Y:=[0,1] \times X \times Y / \sim
$$
}

where the equivalence relation $\sim$ is given by

$$
(0, x, y) \sim\left(0, x, y^{\prime}\right) \text { and }(1, x, y) \sim\left(1, x^{\prime}, y\right),
$$

for any $x, x^{\prime} \in X$ and any $y, y^{\prime} \in Y$. The equivalence class of $(t, x, y)$ is denoted by $(1-t) x \oplus t y$. Given maps $T: X \rightarrow X$ and $S: Y \rightarrow Y$, we defined the map $T * S: X * Y \rightarrow X * Y$ by

$$
T * S((1-t) x \oplus t y)=(1-t) T x \oplus t S y .
$$


A dynamical system $(X, T)$ is said to be fixed-point free it has no fixed point, i.e. $P_{1}(X, T)=\emptyset$. It is said to be aperiodic (or free) if it has no periodic point, i.e. $P_{n}(X, T)=\emptyset$ for all $n \geq 1$.

Let $(X, T)$ be a dynamical system. For each prime number $p$, the pair

$$
\left(P_{p}(X, T), T\right)
$$

is a $\mathbb{Z}_{p}$-space. If $(X, T)$ is a fixed-point free dynamical system then $\left(P_{p}(X, T), T\right)$ becomes a free $\mathbb{Z}_{p^{-}}$-space. The paper [TTY20] investigated its index and proved

Theorem 1.1 ([TTY20], Theorem 1.2). Let $(X, T)$ be a fixed-point free dynamical system. The sequence

$$
\operatorname{ind}_{p} P_{p}(X), \quad(p=2,3,5,7,11, \ldots)
$$

has at most linear growth in p. Namely there exists a positive number $C$ satisfying

$$
\operatorname{ind}_{p} P_{p}(X)<C \cdot p
$$

for all prime numbers $p$.

So the sequence $\operatorname{ind}_{p} P_{p}(X)(p=2,3,5, \ldots)$ cannot be an arbitrary sequence. It has a nontrivial restriction. But, is this restriction optimal? Is there a fixed point free dynamical system $(X, T)$ such that

$$
\operatorname{ind}_{p} P_{p}(X)>C \cdot p
$$

for some positive number $C$ and all sufficiently large prime numbers $p$ ? This is a difficult question because (at least for our current technology) it is hard to estimate $\operatorname{ind}_{p} P_{p}(X)$ from below.

Indeed even the following simpler question has been open.

Problem 1.2 ([TTY20], Problem 7.2). Is there a fixed-point free $d y$ namical system $(X, T)$ such that the sequence

$$
\operatorname{ind}_{p} P_{p}(X), \quad(p=2,3,5,7,11, \cdots)
$$

is unbounded?

The main purpose of this paper is to solve this problem affirmatively.

1.3. Main result. Let $\mathcal{S}=\mathbb{R} / 2 \mathbb{Z}$. Let $\rho$ be a $\mathcal{S}$-invariant metric on $\mathcal{S}$ defined by

$$
\rho(x, y)=\min _{n \in \mathbb{Z}}|x-y-2 n| .
$$

Let $\sigma$ be the (left)-shift on $\mathcal{S}^{\mathbb{Z}}$. Define a subsystem of $\left(\mathcal{S}^{\mathbb{Z}}, \sigma\right)$ by

$\mathcal{Z}:=\left\{\left(x_{n}\right)_{n \in \mathbb{Z}} \in \mathcal{S}^{\mathbb{Z}}: \forall n \in \mathbb{Z}\right.$, either $\rho\left(x_{n-1}, x_{n}\right) \geq \frac{1}{2}$ or $\left.\rho\left(x_{n}, x_{n+1}\right) \geq \frac{1}{2}\right\}$.

Obviously, the dynamical system $(\mathcal{Z}, \sigma)$ has no fixed points and $P_{p}(\mathcal{Z}, \sigma) \neq$ $\emptyset$ for all prime numbers $p$.

Now we state our main result. 
Theorem 1.3. We have

$$
\lim _{p \rightarrow \infty} \operatorname{coind}_{p} P_{p}(\mathcal{Z}, \sigma)=\infty,
$$

where $p$ runs over prime numbers.

Since we know

$$
\operatorname{coind}_{p} P_{p}(\mathcal{Z}, \sigma) \leq \operatorname{ind}_{p} P_{p}(\mathcal{Z}, \sigma)
$$

we also have

$$
\lim _{p \rightarrow \infty} \operatorname{ind}_{p} P_{p}(\mathcal{Z}, \sigma)=\infty .
$$

So this solves Problem 1.2 affirmatively.

We would like to remark that our proof of Theorem 1.3 is noneffective. We cannot figure out the actual growth rate of $\operatorname{coind}_{p} P_{p}(\mathcal{Z}, \sigma)$ from our proof. This remains to be a task for a future study. The main difficulty is that (at least for the authors) it is very hard to directly study the topology of $P_{p}(\mathcal{Z}, \sigma)$. Our proof is indirect and uses the marker property (see §2.2).

Using Theorem 1.3, we can also construct some other fixed-point free dynamical systems having divergent coindex sequence. Let $\rho_{N}$ be a $\mathcal{S}^{N}$-invariant metric on $\mathcal{S}^{N}$ defined by

$$
\rho_{N}\left(\left(x_{i}\right)_{i=1}^{N},\left(x_{i}\right)_{i=1}^{N}\right)=\max _{1 \leq i \leq N} \rho\left(x_{i}, y_{i}\right) .
$$

For a positive integer $N$ and $\delta>0$, we define

$$
\mathcal{X}\left(\mathcal{S}^{N}, 1, \delta\right):=\left\{\left(x_{n}\right)_{n \in \mathbb{Z}} \in\left(\mathcal{S}^{N}\right)^{\mathbb{Z}}: \rho_{N}\left(x_{n}, x_{n+1}\right) \geq \delta, \forall n \in \mathbb{Z}\right\},
$$

This notation might look a bit strange. Its meaning will become clearer in $\S 3$. The system $\mathcal{X}\left(\mathcal{S}^{N}, 1, \delta\right)$ has no fixed point.

Lemma 1.4. We have the following equivariant embeddings:

$$
\mathcal{X}(\mathcal{S}, 1,1 / 2) \hookrightarrow(\mathcal{Z}, \sigma) \hookrightarrow \mathcal{X}\left(\mathcal{S}^{2}, 1,1 / 2\right) \hookrightarrow \mathcal{X}\left(\mathcal{S}^{N}, 1,1 / 2\right),
$$

for all integers $N \geq 2$.

Proof. The embeddings $\mathcal{X}(\mathcal{S}, 1,1 / 2) \hookrightarrow(\mathcal{Z}, \sigma)$ and $\mathcal{X}\left(\mathcal{S}^{2}, 1,1 / 2\right) \hookrightarrow$ $\mathcal{X}\left(\mathcal{S}^{N}, 1,1 / 2\right)$ are canonical for $N \geq 2$. Define $f:(\mathcal{Z}, \sigma) \rightarrow \mathcal{X}\left(\mathcal{S}^{2}, 1,1 / 2\right)$ by $\left(x_{k}\right)_{k \in \mathbb{Z}} \mapsto\left(x_{k}, x_{k+1}\right)_{k \in \mathbb{Z}}$. It is easy to check that $f$ is an equivariant embedding.

Combining Lemma 1.4 and Theorem 1.3, we get that for all integers $N \geq 2$ and $0<\delta<1 / 2$

$$
\lim _{p \rightarrow \infty} \operatorname{coind}_{p} P_{p}\left(\mathcal{X}\left(\mathcal{S}^{N}, 1, \delta\right)\right)=\infty,
$$

where $p$ runs over prime numbers. 


\section{Preliminaries}

2.1. Properties of $\mathbb{Z}_{p}$-coindex. Let $(X, T)$ be a dynamical system. Following [Shi21], for simplifying notations, we define the periodic coindex of $(X, T)$ as

$$
\operatorname{coind}_{p}^{\text {Per }}(X, T)=\operatorname{coind}_{p}\left(P_{p}(X, T), T\right),
$$

for prime numbers $p$. The following lemma is essentially due to [TTY20, Proposition 3.1 ]. See also the proof in [Shi21, Corollary 3.3].

Lemma 2.1. Let $(X, T)$ and $(Y, S)$ be fixed-point free dynamical systems. Let $p$ be a prime number. Then the following properties hold.

(1) If there is an equivariant continuous map $f: X \rightarrow Y$ then $\operatorname{coind}_{p}^{\mathrm{Per}}(X, T) \leq \operatorname{coind}_{p}^{\mathrm{Per}}(Y, S)$.

(2) The system $(X * Y, T * S)$ has no fixed points and $\operatorname{coind}_{p}^{\mathrm{Per}}(X *$ $Y, T * S) \geq \operatorname{coind}_{p}^{\mathrm{Per}}(X, T)+\operatorname{coind}_{p}^{\mathrm{Per}}(Y, S)+1$.

2.2. Marker property. For a dynamical system $(X, T)$, it is said to satisfy the marker property if for each positive integer $N$ there exists an open set $U \subset X$ satisfying that

$$
U \cap T^{-n} U=\emptyset \text { for all } 0<n<N \text { and, } X=\bigcup_{n \in \mathbb{Z}} T^{n} U \text {. }
$$

For example, an extension of an aperiodic minimal system has the marker property. Gutman [Gut15, Theorem 6.1] proved that every finite dimensional aperiodic dynamical system has the marker property. Here a dynamical system $(X, T)$ is said to be finite dimensional if the topological dimension (a.k.a the Lebesgue covering dimension) of $X$ is finite. The marker property has been intensively used in the context of mean dimension theory.

Probably the marker property seems to have nothing to do with the study of $\mathbb{Z}_{p}$-index. But indeed it has.

We can easily see that if a dynamical system has the marker property then it is aperiodic. It had been an open problem for several years whether the converse holds or not. This problem was solved by [TTY20]. They constructed an aperiodic dynamical system which does not have the marker property. The main ingredient of their proof is the $\mathbb{Z}_{p}$-index theory ${ }^{2}$. The first-named author [Shi21] further developed the argument and proved that there exists a finite mean dimensional aperiodic dynamical system which does not have the marker property.

The proof of Theorem 1.3 uses the method developed in [Shi21].

\footnotetext{
${ }^{2}$ This was a main motivation for [TTY20] to study the interaction between $\mathbb{Z}_{p^{-}}$ index theory and topological dynamics.
} 


\section{INVERSE LIMIT OF A FAMILY OF DYNAMICAL SYSTEMS}

In this section, we follow [Shi21, Section 5] and write the results from $(\mathbb{R} / 2 \mathbb{Z})^{\mathbb{Z}}$ to infinite products of a compact metrizable abelian group.

Let $G$ be a compact metrizable abelian group. Then there is a $G$ invariant metric $\rho$ on $G$ which is compatible with its topology ([Str74]), i.e. $\rho(x+g, y+g)=\rho(x, y)$ for any $x, y, g \in G$. Let $\sigma$ be the (left)-shift on $G^{\mathbb{Z}}$, i.e. $\sigma\left(\left(x_{k}\right)_{k \in \mathbb{Z}}\right)=\left(x_{k+1}\right)_{k \in \mathbb{Z}}$. For any positive integers $m$ and any number $\delta>0$, we define a subsystem $(\mathcal{X}(G, m, \delta), \sigma)$ of $\left(G^{\mathbb{Z}}, \sigma\right)$ by

$$
\mathcal{X}(G, m, \delta):=\left\{\left(x_{n}\right)_{n \in \mathbb{Z}} \in G^{\mathbb{Z}}: \rho\left(x_{n}, x_{n+m !}\right) \geq \delta, \forall n \in \mathbb{Z}\right\},
$$

where $m !=m \cdot(m-1) \cdots 2 \cdot 1$. It is clear that $(\mathcal{X}(G, m, \delta), \sigma)$ has no fixed points. We denote by $\left(X_{m}, T_{m}\right):=(\mathcal{X}(G, m, \delta), \sigma)$ for convenience when $G$ is fixed.

For $m>1$, we define an equivariant continuous map $\theta_{m, m-1}$ from $X_{m}$ to $G^{\mathbb{Z}}$ by

$$
\left(x_{k}\right)_{k \in \mathbb{Z}} \mapsto\left(\sum_{i=0}^{m-1} x_{k+i(m-1) !}\right)_{k \in \mathbb{Z}} .
$$

A simple computation shows that

$$
\begin{aligned}
& \rho\left(\sum_{i=0}^{m-1} x_{k+i \cdot(m-1) !}, \sum_{i=0}^{m-1} x_{(k+(m-1) !)+i \cdot(m-1) !}\right) \\
= & \rho\left(\sum_{i=0}^{m-1} x_{k+i \cdot(m-1) !}, \sum_{i=1}^{m} x_{k+i \cdot(m-1) !}\right) \\
= & \rho\left(x_{k}, x_{k+m \cdot(m-1) !}\right)=\rho\left(x_{k}, x_{k+m !}\right), \forall k \in \mathbb{Z} .
\end{aligned}
$$

Then we obtain that the image of $X_{m}$ under $\theta_{m, m-1}$ is contained in $X_{m-1}$. For $m>n$, we define $\theta_{m, n}=\theta_{m, m-1} \circ \theta_{m-1, m-2} \circ \ldots \theta_{n+1, n}$ to be an equivariant continuous map from $X_{m}$ to $X_{n}$.

Fix $a=\left(a_{k}\right)_{k \in \mathbb{Z}} \in G^{\mathbb{Z}}$. For $m \geq 2$, we define a map $\eta_{m-1, m}=\eta_{m-1, m}^{a}$ : $X_{m-1} \rightarrow G^{\mathbb{Z}}$ by $\eta_{m-1, m}\left(\left(x_{k}\right)_{k \in \mathbb{Z}}\right)=\left(y_{k}\right)_{k \in \mathbb{Z}}$ where

$$
y_{k}= \begin{cases}a_{k} & \text { if } 0 \leq k \leq(m-1) \cdot(m-1) !-1, \\ x_{k-(m-1) \cdot(m-1) !}-\sum_{i=1}^{m-1} a_{k-i \cdot(m-1) !} & \text { if }(m-1) \cdot(m-1) ! \leq k \leq m !-1 \\ \sum_{i=0}^{n-1}\left(x_{i \cdot m !+(m-1) !+j}-x_{i \cdot m !+j}\right)+y_{j} & \text { if } k=n \cdot m !+j \\ & \text { with } n>0 \text { and } 0 \leq j \leq m !-1, \\ \sum_{i=n}^{-1}\left(x_{i \cdot m !+j}-x_{i \cdot m !+(m-1) !+j}\right)+y_{j} & \text { if } k=n \cdot m !+j \\ & \text { with } n<0 \text { and } 0 \leq j \leq m !-1 .\end{cases}
$$

Obviously, the map $\eta_{m-1, m}$ is continuous. We remark that the map $\eta_{m-1, m}$ is not equivariant. We show several properties of $\eta_{m-1, m}$ in the following lemmas. 
Lemma 3.1. For $m \geq 2, \eta_{m-1, m}\left(X_{m-1}\right) \subset X_{m}$.

Proof. Let $x \in X_{m-1}$ and $y=\eta_{m-1, m}(x)$. Let $k=n \cdot m !+j$ with $n \in \mathbb{Z}$ and $0 \leq j \leq m !-1$. We divide the proof in the following three cases according to the value of $n$.

Case 1. $n=1$. We have

$$
y_{k}-y_{k-m !}=x_{(m-1) !+j}-x_{j}+y_{j}-y_{j}=x_{(m-1) !+j}-x_{j} .
$$

Since $x \in X_{m-1}$, we have

$$
\rho\left(y_{k}, y_{k-m !}\right)=\rho\left(x_{(m-1) !+j}, x_{j}\right) \geq \delta .
$$

Case 2. $n \geq 2$. A simple computation shows that

$$
\begin{aligned}
& y_{k}-y_{k-m !} \\
= & \sum_{i=0}^{n-1}\left(x_{i \cdot m !+(m-1) !+j}-x_{i \cdot m !+j}\right)-\sum_{i=0}^{n-2}\left(x_{i \cdot m !+(m-1) !+j}-x_{i \cdot m !+j}\right) \\
= & x_{(n-1) m !+(m-1) !+j}-x_{(n-1) \cdot m !+j}=x_{k-m !+(m-1) !}-x_{k-m !} .
\end{aligned}
$$

It follows that

$$
\rho\left(y_{k}, y_{k-m !}\right)=\rho\left(x_{k-m !+(m-1) !}, x_{k-m !}\right) \geq \delta .
$$

Case 3. $n \leq$ 0. Similarly to Case 1 and Case 2, we have

$$
y_{k}-y_{k-m !}=x_{k-m !+(m-1) !}-x_{k-m !},
$$

and consequently $\rho\left(y_{k}, y_{k-m !}\right) \geq \delta$.

To sum up, we conclude that $y \in X_{m}$ and $\eta_{m-1, m}\left(X_{m-1}\right) \subset X_{m}$.

By Lemma 3.1, we see that $\eta_{m-1, m}$ is the map from $X_{m-1}$ to $X_{m}$. Moreover, we show in the following that $\eta_{m-1, m}$ is indeed a right inverse map of $\theta_{m, m-1}$.

Lemma 3.2. $\theta_{m, m-1} \circ \eta_{m-1, m}=i d, \forall m \geq 2$.

Proof. Let $x \in X_{m-1}$ and $y=\eta_{m-1, m}(x)$. Then we have

$$
\theta_{m, m-1} \circ \eta_{m-1, m}(x)=\theta_{m, m-1}(y)=\left(\sum_{i=0}^{m-1} y_{k+i \cdot(m-1) !}\right)_{k \in \mathbb{Z}} .
$$

If $0 \leq k \leq(m-1) !-1$, then

$$
\begin{aligned}
& \sum_{i=0}^{m-1} y_{k+i \cdot(m-1) !} \\
= & \sum_{i=0}^{m-2} a_{k+i \cdot(m-1) !}+\left(x_{k}-\sum_{i=1}^{m-1} a_{k+(m-1-i) \cdot(m-1) !}\right)=x_{k} .
\end{aligned}
$$


If $k=s \cdot m !+t \cdot(m-1) !+j>(m-1)$ ! for $s \geq 0,0 \leq t \leq m-1$ and $0 \leq j \leq(m-1) !-1$, then

$$
\begin{aligned}
& \sum_{i=0}^{m-1} y_{k+i \cdot(m-1) !} \\
= & \sum_{i=t}^{m-1} y_{s \cdot m !+i \cdot(m-1) !+j}+\sum_{i=0}^{t-1} y_{(s+1) m !+i \cdot(m-1) !+j} \\
= & \sum_{i=t}^{m-1} \sum_{\ell=0}^{s-1}\left(x_{\ell \cdot m !+(i+1) \cdot(m-1) !+j}-x_{\ell \cdot m !+i \cdot(m-1) !+j}\right) \\
& +\sum_{i=0}^{t-1} \sum_{\ell=0}^{s}\left(x_{\ell \cdot m !+(i+1) \cdot(m-1) !+j}-x_{\ell \cdot m !+i \cdot(m-1) !+j}\right)+\sum_{i=0}^{m-1} y_{i \cdot(m-1) !+j} \\
= & \sum_{\ell=0}^{s-1} \sum_{i=0}^{m-1}\left(x_{\ell \cdot m !+(i+1) \cdot(m-1) !+j}-x_{\ell \cdot m !+i \cdot(m-1) !+j}\right) \\
& +\sum_{i=0}^{t-1}\left(x_{s \cdot m !+(i+1) \cdot(m-1) !+j}-x_{s \cdot m !+i \cdot(m-1) !+j}\right)+x_{j} \\
= & \sum_{\ell=0}^{s-1}\left(x_{(\ell+1) \cdot m !+j}-x_{\ell \cdot m !+j}\right)+\left(x_{s \cdot m !+t \cdot(m-1) !+j}-x_{s \cdot m !+j}\right)+x_{j} \\
= & x_{s \cdot m !+j}-x_{j}+x_{k}-x_{s \cdot m !+j}+x_{j}=x_{k} .
\end{aligned}
$$

If $k=s \cdot m !+t \cdot(m-1) !+j$ for $s<0,0 \leq t \leq m-1$ and $0 \leq j \leq(m-1)$ ! -1 , then by the similar computation of the case where $s \geq 0$, we have $\sum_{i=0}^{m-1} y_{k+i \cdot(m-1) !}=x_{k}$. This completes the proof.

Corollary 3.3. Let $K$ be a non-negative integer. Then the following properties hold for $m>n$.

(i) The $\operatorname{map} \theta_{m, n}^{*(K+1)}: X_{m}^{*(K+1)} \rightarrow X_{n}^{*(K+1)}$ is equivariant, continuous and surjective.

(ii) The $\operatorname{map} \eta_{n, m}^{*(K+1)}: X_{n}^{*(K+1)} \rightarrow X_{m}^{*(K+1)}$ is equivariant and continuous.

(iii) The map $\eta_{n, m}^{*(K+1)}$ is a continuous right-inverse of $\theta_{m, n}^{*(K+1)}$, i.e. $\theta_{m, n}^{*(K+1)} \circ \eta_{n, m}^{*(K+1)}=\mathrm{id}$.

Proof. By definition of joining of spaces and maps, (i), (ii) and (iii) are clear by the argument in this section.

\section{Proof of Theorem 1.3}

Let $\mathbb{Z}_{3}:=\mathbb{Z} / 3 \mathbb{Z}$ as before. $\mathrm{A} \mathbb{Z}_{3}$-invariant metric $\rho$ on the finite abelian group is $\rho(x, y)=\delta_{0}(x-y)$ where $\delta$ is the Dirac operator, i.e. 
$\delta_{0}(a)=0$ if and only if $a=0$. For $m \geq 1$ and $0<\delta<1$, the subshift $\left(\Sigma_{m}, \sigma\right):=\mathcal{X}\left(\mathbb{Z}_{3}, m, \delta\right)$ of the full shift $\left(\mathbb{Z}_{3}^{\mathbb{Z}}, \sigma\right)$ has the form:

$$
\Sigma_{m}=\left\{\left(x_{n}\right)_{n \in \mathbb{Z}} \in \mathbb{Z}_{3}^{\mathbb{Z}}: x_{n} \neq x_{n+m !}, \forall n \in \mathbb{Z}\right\} .
$$

Lemma 4.1. The dynamical system $\left(\Sigma_{m}, \sigma\right)$ has no fixed point. The set $P_{p}\left(\Sigma_{m}, \sigma\right)$ is nonempty and finite for every prime number $p>m$ !.

Proof. It is obvious that $P_{1}\left(\Sigma_{m}, \sigma\right)=\emptyset$. Let $p$ be a prime number with $p>m$ !. Notice that

$$
P_{p}\left(\Sigma_{m}, \sigma\right)=\left\{\left(x_{i}\right)_{i \in \mathbb{Z}_{p}} \in \mathbb{Z}_{3}^{\mathbb{Z}_{p}}: x_{i} \neq x_{i+m !}, \forall i \in \mathbb{Z}_{p}\right\} .
$$

Since $p>m$ !, we see that $p$ and $m$ ! are coprime. Let $y_{k}=x_{k \cdot m !} \bmod \mathbb{Z}_{p}$. It follows that

$$
P_{p}\left(\Sigma_{m}, \sigma\right) \cong\left\{\left(y_{k}\right)_{k \in \mathbb{Z}_{p}} \in \mathbb{Z}_{3}^{\mathbb{Z}_{p}}: y_{k} \neq y_{k+1}, k \in \mathbb{Z}_{p}\right\} .
$$

It is easy to check that the right-hand side set is nonempty and finite (see also [TTY20, Lemma 4.1]).

Lemma 4.2. Let $K \geq 0$. Then $\operatorname{coind}_{p}^{\mathrm{Per}}\left(\Sigma_{m}^{*(K+1)}, \sigma^{*(K+1)}\right)=K$ for all prime numbers $p>m$ !.

Proof. By Lemma 4.1, we get that $P_{p}\left(\Sigma_{m}^{*(K+1)}, \sigma^{*(K+1)}\right)=P_{p}\left(\Sigma_{m}, \sigma\right)^{*(K+1)}$ which is an $E_{K} \mathbb{Z}_{p}$-space for any prime number $p>m$ !. Thus we have $\operatorname{coind}_{p} P_{p}\left(\Sigma_{m}^{*(K+1)}, \sigma^{*(K+1)}\right)=K$. This completes the proof.

Let $\mathcal{S}=\mathbb{R} / 2 \mathbb{Z}$. Let $\rho$ be a $\mathcal{S}$-invariant metric on $\mathcal{S}$ defined by

$$
\rho(x, y)=\min _{n \in \mathbb{Z}}|x-y-2 n| .
$$

Define

$\mathcal{Y}:=\left\{\left(x_{n}\right)_{n \in \mathbb{Z}} \in \mathcal{S}^{\mathbb{Z}}: \forall n \in \mathbb{Z}\right.$, either $\rho\left(x_{n-1}, x_{n}\right)=1$ or $\left.\rho\left(x_{n}, x_{n+1}\right)=1\right\}$.

This system is related to the marker property by the next lemma.

Lemma 4.3 ([TTY20], Lemma 5.3). Let $(X, T)$ be a dynamical system having marker property. Then there is an equivariant continuous map from $(X, T)$ to $(\mathcal{Y}, \sigma)$.

We recall the definition of the dynamical system $\mathcal{Z}$. It is a subsystem of $\left(\mathcal{S}^{\mathbb{Z}}, \sigma\right)$ defined by

$\mathcal{Z}:=\left\{\left(x_{n}\right)_{n \in \mathbb{Z}} \in \mathcal{S}^{\mathbb{Z}}: \forall n \in \mathbb{Z}\right.$, either $\rho\left(x_{n-1}, x_{n}\right) \geq \frac{1}{2}$ or $\left.\rho\left(x_{n}, x_{n+1}\right) \geq \frac{1}{2}\right\}$.

The dynamical system $(\mathcal{Z}, \sigma)$ has no fixed points and $P_{p}(\mathcal{Z}, \sigma) \neq \emptyset$ for all prime numbers $p$.

The following proposition is essentially due to [Shi21, Lemma 7.5]. 
Proposition 4.4. Let $(X, T)$ be the inverse limit of a family of dynamical systems $\left\{\left(X_{n}, T_{n}\right)\right\}_{n \in \mathbb{N}}$ via $\tau=\left(\tau_{m, n}\right)_{m, n \in \mathbb{N}, m>n}$ where $\tau_{m, n}: X_{m} \rightarrow$ $X_{n}$ are equivariant continuous maps. Suppose there is a continuous right-inverse $\gamma=\left(\gamma_{n, m}\right)_{n, m \in \mathbb{N}, m>n}$, i.e. $\gamma_{n, m}: X_{n} \rightarrow X_{m}$ are continuous maps with $\tau_{m, n} \circ \gamma_{n, m}=\mathrm{id}$ for $m>n$. If there is an equivariant continuous map $f:(X, T) \rightarrow(\mathcal{Y}, \sigma)$, then there exists an integer $M$ and an equivariant continuous map

$$
g:\left(X_{M}, T_{M}\right) \longrightarrow(\mathcal{Z}, \sigma) .
$$

Proof. Let $\pi_{m}: \mathcal{X} \rightarrow X_{m}$ be the natural projection for $m \in \mathbb{N}$. Let $P_{1}: \mathcal{S}^{\mathbb{Z}} \rightarrow \mathcal{S}$ be the projection on 0-th coordinate. Define $\phi=P_{1} \circ f:$ $X \rightarrow \mathcal{S}$. Then $f(x)=\left(\phi\left(T^{n} x\right)\right)_{n \in \mathbb{Z}}$ for any $x \in X$. Notice that there exists an integer $M>0$ such that

$$
\pi_{M}(x)=\pi_{M}(y) \Longrightarrow \rho(\phi(x), \phi(y))<\frac{1}{4} .
$$

For $m \geq 1$, we define a continuous map $\gamma_{m}: X_{m} \rightarrow X$ by

$$
x \mapsto\left(\tau_{m, 1}(x), \tau_{m, 2}(x), \ldots, \tau_{m, m-1}(x), x, \gamma_{m, m+1}(x), \gamma_{m, m+2}(x), \ldots\right) .
$$

Define a continuous map $\varphi=\phi \circ \gamma_{M}: X_{M} \rightarrow \mathcal{S}$ and an equivariant continuous map $g: X_{M} \rightarrow \mathcal{S}^{\mathbb{Z}}$ by

$$
x \mapsto\left(\varphi\left(T_{M}^{n}(x)\right)\right)_{n \in \mathbb{Z}} .
$$

Since $\pi_{M} \circ \gamma_{M}=\mathrm{id}$ and $\pi_{M} \circ T=T_{M} \circ \pi_{M}$, it follows from (4.1) that

$$
\rho\left(\phi\left(\gamma_{M}\left(T_{M}^{n}(x)\right)\right), \phi\left(T^{n}\left(\gamma_{M}(x)\right)\right)\right)<\frac{1}{4}, \forall n \in \mathbb{Z} .
$$

Fix $x \in X_{M}$ and $n \in \mathbb{Z}$. By definitions of $\mathcal{Y}$ and $f$, there exists an $i \in\{0,1\}$ such that

$$
\rho\left(\phi\left(T^{n+i}\left(\gamma_{M}(x)\right)\right), \phi\left(T^{n+i+1}\left(\gamma_{M}(x)\right)\right)\right)=1 .
$$

Combing $(4 \cdot 3)$ with $(4 \cdot 2)$, we obtain that

$$
\begin{aligned}
& \rho\left(\phi\left(\gamma_{M}\left(T_{M}^{n+i} x\right)\right), \phi\left(\gamma_{M}\left(T_{M}^{n+i+1} x\right)\right)\right) \\
\geq & \rho\left(\phi\left(T^{n+i}\left(\gamma_{M}(x)\right)\right), \phi\left(T^{n+i+1}\left(\gamma_{M}(x)\right)\right)\right) \\
& -\rho\left(\phi\left(\gamma_{M}\left(T_{M}^{n+i} x\right)\right), \phi\left(T^{n+i}\left(\gamma_{M}(x)\right)\right)\right) \\
& \quad-\rho\left(\phi\left(\gamma_{M}\left(T_{M}^{n+i+1} x\right)\right), \phi\left(T^{n+i+1}\left(\gamma_{M}(x)\right)\right)\right) \\
\geq & 1-\frac{1}{4}-\frac{1}{4}=\frac{1}{2} .
\end{aligned}
$$

Since $\varphi=\phi \circ \gamma_{M}$, we have that

$$
\rho\left(\varphi\left(T_{M}^{n+i} x\right), \varphi\left(T_{M}^{n+i+1} x\right)\right) \geq \frac{1}{2} .
$$

By definition of $g$ and arbitrariness of $n$ and $x$, we conclude that the image of $X_{M}$ under $g$ is contained in $\mathcal{Z}$. This completes the proof.

Now we present the proof of our main result. 
Proof of Theorem 1.3. Let $K \geq 0$. By Corollary 3.3 (1), let $(X, T)$ be the inverse limit of the family $\left\{\left(\Sigma_{n}^{*(K+1)}, \sigma^{*(K+1)}\right)\right\}_{n \in \mathbb{N}}$ via $\theta=\left(\theta_{m, n}^{*(K+1)}\right)_{m, n \in \mathbb{N}, m>n}$. Since for every $m \geq 1$,

$$
P_{m !}\left(\Sigma_{m}^{*(K+1)}, \sigma^{*(K+1)}\right)=P_{m !}\left(\Sigma_{m}, \sigma\right)^{*(K+1)}=\emptyset,
$$

we see that $(X, T)$ is aperiodic. Since $\Sigma_{m}$ is 0 -dimensional, we have that $\Sigma_{m}^{*(K+1)}$ is at most of dimension $K$ for any $m \geq 1$ and consequently $X$ is at most of dimension $K$ ([NK70, Section 6]). Since an aperiodic finite dimensional dynamical system has the marker property ([Gut15, Theorem 6.1]), the dynamical system $(X, T)$ has the marker property. By Lemma 4.3, there is an equivariant continuous map from $(X, T)$ to $(\mathcal{Y}, \sigma)$. It follows from Corollary 3.3 and Proposition 4.4 that there exists an integer $M$ and an equivariant continuous map from $\left.\left(\Sigma_{M}^{*(K+1)}, \sigma^{*(K+1)}\right)\right)$ to $(\mathcal{Z}, \sigma)$. By Lemma 2.1, we have

$$
\operatorname{coind}_{p}^{\text {Per }}(\mathcal{Z}, \sigma) \geq \operatorname{coind}_{p}^{\text {Per }}\left(\Sigma_{M}^{*(K+1)}, \sigma^{*(K+1)}\right)=K,
$$

for all prime numbers $p>M$ !. Since $K$ is chosen arbitrarily, we conclude that

$$
\lim _{p \rightarrow \infty} \operatorname{coind}_{p}^{\text {Per }}(\mathcal{Z}, \sigma)=\infty .
$$

\section{REFERENCES}

[Gut15] Yonatan Gutman. Mean dimension and Jaworski-type theorems. Proceedings of the London Mathematical Society, 111(4):831-850, 2015.

[Mat03] Jiří Matoušek. Using the Borsuk-Ulam theorem: lectures on topological methods in combinatorics and geometry. Springer Science \& Business Media, 2003.

[NK70] Keiô Nagami and Yukihiro Kodama. Dimension theory. Academic Press, 1970.

[Shi21] Ruxi Shi. Finite mean dimension and marker property. arXiv preprint arXiv:2102.12197, 2021.

[Str74] Raimond A Struble. Metrics in locally compact groups. Compositio Mathematica, 28(3):217-222, 1974.

[TTY20] Masaki Tsukamoto, Mitsunobu Tsutaya, and Masahiko Yoshinaga. $G$-index, topological dynamics and marker property. arXiv preprint arXiv:2012.15372, 2020.

Institute of Mathematics, Polish Academy of Sciences, ul. Śniadeckich

8, 00-656 Warszawa, Poland

Email address: rshi@impan.pl

Department of Mathematics, Kyushu University, Moto-oka 744, NishiKU, FUKUOKA 819-0395, JAPAN

Email address: tsukamoto@math.kyushu-u.ac.jp 